\title{
RELAÇÃO DE AJ UDA NO DESEMPENHO DOS CUIDADOS DE ENFERMAGEM A DOENTES EM FIM DE VIDA
}

\author{
Helping relationship in end-of-life patient's nursing care context \\ Relacíon de ayuda en el contexto de los cuidados de enfermeria a enfermos al final \\ de su vida
}

Rosa Maria Pereira Simões

Manuel Alves Rodrigues ${ }^{2}$

\begin{abstract}
RESUMO
Este estudo tem como principal objetivo verificar a importância que os enfermeiros atribuem às competências relacionais na ajuda a doentes em fim de vida. A investigação é de tipo descritivo-correlacional, transversal, com uma amostra de 56 enfermeiros, selecionada de forma intencional, acidental e desenvolvida a partir da aplicação de um instrumento de medida que integra 0 Inventário de Competências Relacionais de Ajuda, Escala de Satisfação com a Vida, Questões relativas à importância atribuída à formação contínua. Da análise dos resultados ressalta-se que os enfermeiros consideram as competências relacionais fundamentais para o cuidado ao doente em fim de vida. 0 desempenho de competências relacionais de ajuda está significativamente correlacionado com a formação que os enfermeiros desenvolveram sobre relação de ajuda, bem como com a satisfação que têm com a vida em geral.
\end{abstract}

Palavras-chave: Enfermagem. Cuidados de Enfermagem. Doente Terminal. Relações Interpessoais. Educação Continuada

\begin{abstract}
This study's main objective is to verify the importance that nurses attach to relational skills, in helping end of life patients. The research is descriptive-correlational, cross-sectional with a sample of 56 nurses selected by an intentional, accidental and developed from the application of a measuring instrument that integrates the Helping Relational Skills Inventory, Life Satisfaction Scale, and questions concerning the importance attached to continuous training. The results analysis highlight that nurses consider the relational skills essential to end of life patient care. The results indicate that the best relational skills nurses' performance is correlated with better training in helping relationships and also with the perception of greater satisfaction with life in general. These results have application in personal and professional nurse's skills developing process.
\end{abstract}

Keywords: Nursing. Nursing care. Terminally ill. Interpersonal relations. Education, continuing

\section{Resumen}

Este estudio tiene como principal objetivo comprobar la importancia que conceden los enfermeros a las competencias de relacionamiento, en la ayuda a enfermos al final de su vida. La investigación es de tipo descriptivo y de correlación, transversal, con una muestra de 56 enfermeros, seleccionada de forma intencional, accidental y desarrollada a partir de la aplicación de un instrumento de medida integrado en el Inventario de competencias relacionales de ayuda, Escala de satisfacción con la vida, cuestiones referentes a la importancia concedida a la formación continua. Del análisis de los resultados concluimos que los enfermeros consideran las competencias de relacionamiento fundamentales para cuidar al enfermo al final de su vida. Los resultados indican que el mejor resultado de competencias de relacionamiento, por parte de los enfermeros, está correlacionado con mejor formación sobre relación de ayuda, así como la percepción de mayor satisfacción con la vida en general. Estos resultados tienen aplicación en el proceso de desarrollo de competencias personales y profesionales de los enfermeros.

Palabras Clave: Enfermería. Atención de enfermería. Enfermo terminal. Relaciones interpersonales. Educación continua

'Mestre em Ciências de Enfermagem, Enfermeira Graduada, Hospitais da Universidade de Coimbra, Serviço de Urgência,Coimbra,Distrito de Coimbra, Portugal. E-mail: rosasimoes18@gmail.com,2Ph.D, Agregação. Professor Coordenador com Agregação, Escola Superior de Enfermagem de Coimbra,Coimbra, Distrito de Coimbra.Portugal. Unidade de Investigação em Ciências da Saúde: Enfermagem. E-mail: demar7@gmail.com 


\section{INTRODUÇÃO}

A relação de ajuda, enquanto intervenção autônoma, é um elemento decisivo e inquestionável na atividade dos enfermeiros e desempenha papel central na resposta às necessidades concretas de cada pessoa, independentemente da sua situação/problema ou contexto em que se desenrola a ação. No entanto, os cuidados de enfermagem, apoiados na técnica de relação de ajuda, são particularmente complexos, em situações de dependência, situações de medo, dor extrema, solidão e abandono. No caso concreto dos doentes em fase terminal de vida, o enfermeiro necessita de recorrer a estratégias terapêuticas planeadas e a um sistema integrado de competências relacionais, aproximando-se dos espaços de intimidade, demonstrando competências de contato e competências sociais empáticas. Coloca-se então a questão: quais são as competências relacionais que os enfermeiros mais valorizam no cuidado que prestam aos doentes em cuidados paliativos?

A promoção de competências relacionais por meio de estratégias de formação, emerge assim como uma necessidade fundamental ao longo da vida, pelo que definimos como um objetivo de estudo, de analisar a relação entre competências relacionais e formação contínua. Porém, mais formação nem sempre significa mais eficácia prática, uma vez que a ação está relacionada com o sujeito que a pratica. Os estados emocionais e motivacionais positivos são condição necessária para uma ação empenhada em contextos complexos. A autoestima e a satisfação com a vida em geral é condição para que o enfermeiro se envolva com entusiasmo e eficácia no seu meio social e no seu trabalho. Para cuidarem dos outros, os enfermeiros devem poder ter uma avaliação subjetiva, positiva, de si mesmo. Neste sentido, é também objetivo deste estudo analisar a relação entre competências relacionais e satisfação dos enfermeiros com a vida em geral. Desenvolvemos uma investigação que indaga sobre as competências relacionais de ajuda que os enfermeiros valorizam no plano de cuidados a doentes em fim de vida e procuramos, ainda, compreender em que medida a formação em relação de ajuda e a satisfação com a vida em geral são fatores que se correlacionam com as diferentes dimensões das competências relacionais. Depois de uma primeira parte de enquadramento teórico, desenvolvemos a parte empírica do processo de investigação.

\section{RELAÇÃO DE AJ UDA NA ARTE DE CUIDAR}

Entre muitas autoras de enfermagem, Jean Watson valoriza a arte de cuidar como um ideal moral, mais do que um comportamento orientado pela tarefa e inclui características, como a ocasião do cuidar real e o momento do cuidar transpessoal, fenômenos que ocorrem quando existe um verdadeiro relacionamento enfermeiro-doente ${ }^{1}$. Para autora, a relação de ajuda é a essência, meio poderoso de intervenção e um elemento decisivo e inquestionável no cuidar em Enfermagem. 0 enfermeiro distingue-se pela formação e experiência que lhe permite entender e respeitar os outros, em um quadro onde procura abster-se de juízos de valor relativamente à pessoa cliente dos cuidados de Enfermagem². Para Phaneuf ${ }^{3}$, a relação de ajuda é uma técnica particularmente significativa, dado que a pessoa que ajuda está completamente voltada para o outro, para a sua situação e para o seu sofrimento, definindo-a como "uma troca tanto verbal como não verbal que ultrapassa a superficialidade e que favorece a criação do clima de compreensão e apoio de que a pessoa tem necessidade, no decurso de uma prova"3:324. A pessoa que ajuda tem sempre como objetivo promover no outro o crescimento pessoal, o desenvolvimento, a maturidade, um melhor funcionamento e uma maior capacidade de enfrentar as mudanças que ocorrem na vida. Existe, no entanto, uma questão importante que deve ser clarificada e que justifica a intencionalidade de tornar um encontro terapêutico. Responder com benevolência, querer ser útil, colocar o doente à vontade, ser simpático, são ações que, por mais úteis e necessárias que sejam, apenas respondem a imperativos habituais de cuidados; nenhuma destas ações responde ao apelo de ajuda feito em situações emocionalmente carregadas de sofrimento ou de indecisão. ${ }^{3}$ As ações que visam apenas o bem-estar físico não podem ser confundidas com relação de ajuda; no entanto, podem tornar-se esse tipo se forem seguidas de uma intervenção que vise o reconforto afetivo, a satisfação das suas necessidades ou a resolução de um problema existencial. 0 poder da relação de ajuda é particularmente complexa, no plano dos cuidados ao doente em fim de vida, sendo vários os fatores que interferem no processo de interacção enfermeirodoente, quando cuidam de doentes terminais, como referem Sapeta e Lopes. ${ }^{4} 0$ enfermeiro partilha diariamente de uma variedade de sentimentos acerca do sofrimento, uma vez que a doença terminal condiciona a pessoa a perdas sucessivas, colocando em perigo o seu equilíbrio. Estas perdas surgem inicialmente da própria doença (sintomas, internamentos, tratamentos, recaídas ou ausência de cura), e ocorrem também perda do papel social (ruptura com o meio profissional, social e familiar), levando a uma perda da identidade e referências, sentidas como uma decadência e vividas com uma enorme angústia. As reações emocionais a doenças graves são complexas de lidar, ${ }^{5}$ pelo que o enfermeiro precisa de aprender a construir a relação na sua prática de cuidados, no contexto de emoções intensas. ${ }^{6}$ Para Haya et al.. os diagnósticos de enfermagem prevalentes em cuidados paliativos são: a fadiga, o medo, o sentimento de impotência, a angústia espiritual, ansiedade, a perturbação da imagem corporal, a dor e 0 sofrimento (luto). Estes autores consideram que as ações de suporte de enfermagem visam reforçar as estratégias de adaptação e devem apoiar-se no suporte social (informativo, 
emocional, autoestima e organizacional), nas estratégias de "coping" e no conceito de saúde incluído na natureza dos cuidados de enfermagem.

\section{COMPETÊNCIAS RELACIONAIS DE AJ UDA NO CONTEXTO DO CUIDADO AO DOENTE EM FIM DE VIDA}

0 complexo cotidiano de trabalho, com doentes em sofrimento profundo, implica que o enfermeiro regule emoções, expresse sentimentos de entusiasmo, manifeste zelo e confiança, capacidade de dedicação ao trabalho, tenacidade e persistência, raciocínio analítico, capacidade relacional e de comunicação, auto-organização, autoconfiança, autocontrolo, autoeficácia, curiosidade, prazer em aprender, compreensão das emoções e dos sentimentos, capacidade de decisão, de partilha e de cooperação. ${ }^{8} 0$ modelo de descrição e agrupamento do conteúdo das competências desenvolvido por Dias $^{8}$ organiza as competências em categorias multidimensionais, centradas nas dimensões pessoal, científica, técnica, socioafetiva, relacional, comunicativa e ético-moral. Esta autora propõe uma tipologia de competências que agrupa todos os saberes, que devem ser desenvolvidos ao longo do processo formativo para que os formandos se desenvolvam ao nível do saber (competências do domínio cognitivo), do saberfazer (competências de domínio psicomotor) e do saber-ser (competências do domínio socioafectivo, relacional e ético moral). A investigação de Ferreira, Tavares e Duarte conclui que "da mesma forma que procuramos conhecimentos e habilidades para o desenvolvimento de técnicas de enfermagem para os cuidados físicos, também se deve ter a mesma preocupação em relação às habilidades designadas por comportamentos e competências ligados às funç̃̃es de contato", 9:53 referindo-se ao toque, ao olhar, às distâncias, às posições físicas, ao tempo, à escuta e aos meios de comunicação. Os autores propõem o seguinte modelo de classificação das competências relacionais: genéricas, empáticas, comunicação e contato. 0 desenvolvimento destas competências é fundamental no exercício da Enfermagem. Nas orientações atuais das politicas de Ensino Superior na Europa, a aprendizagem deve centrar-se em competências, e este princípio deve aplicar-se ao processo de formação dos enfermeiros, no quadro de desenvolvimento de competências pessoais, sociais e profissionais, com relevo para a relação de ajuda empática. Adam, Queiroz e Sousa ${ }^{10-12}$ consideram que um programa formativo sustentado é fundamental quer para o estabelecimento da relação terapêutica enfermeiro-doente, quer para o desenvolvimento pessoal do enfermeiro. Assumindo-se que a formação contínua é um aspecto fundamental para a qualidade dos cuidados ao doente em fim de vida e sendo as competências relacionais de ajuda integrantes e essenciais nestes cuidados, faz todo o sentido que haja, por parte dos enfermeiros e das instituições, um investimento na formação contínua nestas áreas de intervenção. Quando elaboram os programas de formação, os responsáveis das equipes devem ter presente um conjunto dinâmico de variáveis: por um lado as diversas dimensões das competências relacionais que é preciso melhorar; em segundo lugar, a incerteza e imprevisibilidade que caracteriza o contexto de cuidados a doentes em fim de vida; em terceiro lugar, a importância da regulaçã̃o dos fatores que influenciam o bem-estar e a saúde em geral dos enfermeiros que trabalham nesses contextos.

\section{METODOLOGIA}

\section{Tipo de Estudo}

Estudo descritivo-correlacional, transversal, com os seguintes objetivos: Identificar o valor atribuído pelos enfermeiros às competências relacionais de ajuda na sua prática de cuidados ao doente em fim de vida; analisar a relação entre o valor atribuído pelos enfermeiros às competências relacionais de ajuda e a variável importância atribuída à formação contínua em relação de ajuda; e verificar se o valor atribuído às competências relacionais de ajuda na prática de cuidados ao doente em fim de vida é maior nos enfermeiros que apresentam maior valor de satisfação com a vida em geral.

\section{Hipóteses}

Existe relação entre as dimensões das competências relacionais de ajuda (genéricas, empáticas, comunicação, contato), a formação contínua em relação de ajuda e a satisfação com a vida em geral.

\section{População/amostra}

Da população de enfermeiros a trabalhar em serviços de cuidados terminais, na Região Centro de Portugal, selecionou-se uma amostra de 56 enfermeiros. 0 número de serviços que prestam cuidados paliativos na Região Centro é ainda limitado, pelo que empregam ainda uma pequena população de cuidadores, o que explica o relativo tamanho reduzido da amostra. Como critério de inclusão, estabeleceuse que os enfermeiros deveriam trabalhar há mais de um ano na Unidade de Cuidados Paliativos. A técnica de amostragem foi intencional e acidental, com presença do investigador nos locais de trabalho, em dias previamente planejados com as equipes, para não perturbar o normal funcionamento dos cuidados.

\section{Instrumento de recolha de dados}

Utilizamos um inventário de Competências Relacionais de Ajuda, da autoria de Ferreira, Tavares e Duarte ${ }^{9}$, com boa consistência interna (alfa $=0,81$ ). São analisadas quatro dimensões na variável competências relacionais (genéricas, empáticas, comunicação, contato). Este inventário, na sua versão final, é constituído por 51 itens. Esta escala é de tipo Likert, em sete níveis, sendo um a máxima discordância e sete a máxima concordância. Quanto maior for a pontuação obtida, maior o grau de competências relacionais. A pontuação global pode variar de um mínimo de 51 a um máximo de 357. A Escala de Satisfação com a Vida, elaborada por Diener et al. ${ }^{13}$, 
é unidimensional com boa consistência interna (alfa $=0,83$ ); avalia o juízo subjetivo que cada indivíduo faz sobre a qualidade da própria vida, de acordo com critérios estabelecidos por si, e não em função de padrões impostos externamente, pelo investigador ou por outrem. Utilizamos uma versão composta por 5 itens formulados no sentido positivo, pertencentes a uma escala tipo Likert de 5 pontos, podendo a pontuação variar entre um mínimo de 5 e um máximo de 25 pontos. 0 questionário integra questões relativas à importância atribuída à Formação Contínua em Relação de Ajuda, realizadas durante os últimos dois anos de exercício profissional. As questões sobre a formação contínua em relação de ajuda são do tipo fechadas e referentes ao tipo de formação realizada e à importância atribuída a essa mesma formação, quer nos contributos para o aumento dos conhecimentos sobre relação de ajuda, na melhoria das competências ao cuidar do doente em fim de vida ou na melhoria das relações de ajuda entre os elementos da equipe. Foram formuladas a partir da literatura.

\section{Aspectos éticos}

Durante o processo de investigação foram respeitados todos os princípios éticos e legais, seguindo um fluxograma de princípios: pedido de autorização aos autores dos instrumentos de medida, pedido de autorização às direções dos hospitais para colher os dados, autorização expressa dos enfermeiros que voluntariamente assumiram pertencer à amostra, aconselhamento de peritos em bioética.

\section{RESULTADOS}

Caracterização da amostra: Constituída por 13 enfermeiros (23,2\%) e 43 enfermeiras (76,8\%). Destes, 34 enfermeiros (60,7\%), 17 enfermeiros graduados $(30,4 \%), 3$ enfermeiros especialistas (5,4\%), 2 enfermeiros chefes $(3,6 \%)$. A idade média foi de $31,44(d p=7,91)$. Quanto ao tempo de serviço, a média foi de 5,62 ( $d p=4,91)$.

Competências relacionais de ajuda: Procedemos ao cálculo das pontuações obtidas nas respostas ao inventário de Competências Relacionais de Ajuda e verificaram-se os seguinte resultados: para o peso global da escala, houve média de 281,07 em um intervalo de 51 a 357 , com um desvio-padrão de 29,33, valores que correspondem a uma média ponderada de 5,51 em uma escala que varia entre mínimo 1 e máximo 7; na dimensão competências de contato, observou-se uma média ponderada de 4,78 e, nas competências genéricas, média ponderada de 5,96. Não se observaram diferenças entre 0 sexo masculino e o sexo feminino.

Importância da formação contínua: No que se refere à análise da variável importância atribuída à formação contínua em "relação de ajuda": Para aumentar conhecimentos (média $=3,66, \mathrm{dp}=0,85$ ); Para melhorar as competências (média $=3,78, \mathrm{dp}=0,94)$; Para melhorar a relação na equipe ( édia $=3,57, \mathrm{dp}=0,98$ )
Satisfação com a vida em geral: A média foi de 18,66 ( $\mathrm{dp}=3,89)$, considerando uma pontuação mínima de 8 e máxima de 24.

Relação entre competências relacionais e formação contínua: 0 recurso a medidas paramétricas foi ponderado em função dos resultados do teste de normalidade Kolmogoriv-Serminov $(z=0,07, p=0,20)$. Considerando $p>0,05$, observa-se que existe uma correlação estatisticamente significativa entre 0 peso global do valor atribuído às competências relacionais de ajuda e a importância atribuída à formação contínua em relação de ajuda: Competências genéricas $(r=0,31, p=0,01)$; empáticas $(r=0,28, p=0,03)$; comunicação $(r=0,20, p=0,03)$; contato $(r=0,30, p=0,02)$, total $(r=0,35, p=0,01)$.

Relação entre competências relacionais e satisfação com a vida: Considerando $p>0,05$, observa-se uma correlação positiva entre o valor atribuído às competências relacionais de ajuda e o nível de satisfação com a vida em geral: Genéricas $(r=0,31, p=0,01)$, empáticas $(r=0,25, p=0,31)$, comunicação $(r=0,29, p=0,034)$, contato $(r=0,13, p=0,34)$, total $(r=0,41, p=0,01)$.

\section{DISCUSSÃO}

Trata-se de uma amostra de enfermeiros relativamente jovem, com média de 31,44 anos, mas já com alguma experiência de prestação de serviço, em cuidados continuados. No quadro de competências não se observam diferenças entre os enfermeiros e as enfermeiras, sendo a dimensão de competências genéricas as mais pontuadas (média ponderada de 5,96) e as de contato, as menos pontuadas (média ponderada de 4,78). Os valores obtidos para a dimensão competências de contato podem ser compreendidos recorrendo a Chalifour ${ }^{14}$ que afirma que a utilização de maiores distâncias pelos enfermeiros é muitas vezes uma forma de proteção pessoal e uma fuga à intimidade com o doente em estado crítico, mantendo-se uma relação superficial de não comprometimento. Por outro lado, a maior valorização das competências genéricas, por parte dos enfermeiros, no contexto do cuidado ao doente em fim de vida, pode ser entendida como uma interiorização de um modelo teórico em que o doente é tomado como parceiro dos cuidados, colaborando na tomada de decisão sobre os cuidados necessários para a promoção da sua saúde, restabelecimento da mesma ou morte serena. ${ }^{14}$. No que se refere à análise da variável, importância atribuída à formação contínua em relação de ajuda, a média é superior na perspectiva de melhorar as competências de cuidar o doente em fim de vida (3,78), embora não se afaste muito da perspectiva de melhoria de conhecimentos ou favorecimento da relação entre os elementos da equipe. A formação em relação de ajuda é um fator que os enfermeiros indicam que pode ser melhorado. Os enfermeiros parecem conscientes da necessidade imperiosa de dar resposta aos problemas complexos do doente em fim de vida. Segundo 
Watson ${ }^{1}$, para ir ao encontro das necessidades dos doentes, a formação em Enfermagem deve proporcionar a mudança do saber-fazer para o saber ser, com formas de conhecimento baseadas em valores humanos e no interesse pelos outros. De acordo com os resultados, a formação deve enfatizar as competências empáticas e as competências corporais de contato. A hipótese formulada da correlação entre competências de ajuda e formação confirmou-se $(r=0,35, p=0,01)$. As correlações foram significativas na dimensão contato, genéricas e empáticas, o que coloca estes conceitos em uma posição central, no processo de formação contínua dos enfermeiros. 0 investimento na formação contínua em enfermagem ${ }^{12}$ deve ser contemplado no projeto estratégico de melhoria dos serviços e alargado a outros tipos de formação, nomeadamente por meio da elaboração de projetos de investigação no local de trabalho, promoção de encontros temáticos como lugares privilegiados para o debate e reflexão e incentivo à formação pós-graduada. 0 enfermeiro chefe, enquanto agente de mudanças, assume papel fundamental junto da equipe, criando estratégias de motivação e de compensação pelos esforços dispendidos pelos seus colaboradores, devendo ainda assumir o papel de mediador, na articulação com as estruturas de formação. Um importante resultado foi encontrado ao confirmar-se a hipótese de correlação entre competências e satisfação de vida $(r=0,41$, $p=0,01$ ). Diener e Suh ${ }^{15}$ concluíram que a satisfação com a vida em geral se correlaciona de forma significativa com o desempenho em contexto de trabalho, que apresenta valores mais elevados quanto mais elevada for a satisfação em geral.

\section{CONCLUSÕES}

Em função dos resultados discutidos, podemos concluir que os enfermeiros nos seus serviços estão atentos à complexa problemática de cuidar de doentes em fim de vida. Para cuidar destes doentes, as competências relacionais de ajuda assumem um papel central e determinante. Este estudo indica que os enfermeiros necessitam de melhorar, continuamente, as diversas dimensões da relação da ajuda, especialmente no uso do corpo como ferramenta terapêutica, por meio do tato e na promoção da compreensão empática. A formação contínua desempenha um papel fundamental da promoção de competências relacionais aplicadas à melhoria dos cuidados eà boa relação entre os elementos da equipe, pelo que deve ser programada e assumida como missão estratégica de um serviço. Os enfermeiros cuidam em condições difíceis, sobretudo quando se trata de doentes em fim de vida, para isso necessitam de um meio favorável que lhes permita ter uma percepção positiva do trabalho, ampliando assim a sua avaliação positiva da vida em geral. Embora este estudo tenha limitações, no que concerne ao poder de generalização dos resultados, dá-nos indicações muito úteis para a definição de um perfil de competências relacionais adequadas ao cuidados de doentes em sofrimento profundo e indicações para os cuidados a ter no trabalho com a satisfação pessoal. No sentido de continuar a contribuir para a análise do tema, parece-nos pertinente a realização de estudo com maior número de elementos na amostra e inclusão em estudos futuros, de outras variáveis, como sejam, o clima organizacional, liderança, satisfação no trabalho e satisfação dos utilizadores dos serviços.

\section{REFERÊNCIAS}

1. Watson J. Nursing: the philosophy and science of caring. $2^{\mathrm{a}}$ ed. Boulder, Colorado (USA): Associated University Press; 1985.

2. Ordem dos Enfermeiros (PT). Padrões de qualidade dos cuidados de enfermagem. Lisboa (PT): Divulgar; 2001.

3. Phaneuf M. Comunicação, entrevista, relação de ajuda e validação. Loures (PT): Lusociência; 2005.

4. Sapteta P, Lopes M. Cuidar em fim de vida: factores que interferem no processo de interacção enfermeiro-doente. Referência: revista de educação e formação em enfermagem 2007 jun; II (4); 35-57.

5. Marques AR, et al. Reacções emocionais à doença grave: como lidar...Coimbra (PT): Psiquiatria Clínica; 1991.

6. Frias C. 0 enfermeiro com a pessoa em fim de vida na construção do cuidar. Referência: revista de educação e formação em enfermagem 2008 jun; II (6); 57-67.

7. Ahya P, et al. Desafios da enfermagem em cuidados paliativos: "cuidar": ética e práticas. Loures (PT): Lusociência; 2000.

8. Dias M. Construção e validação de um inventário de competências: contributos para a definição de um perfil de competências do enfermeiro com o grau de licenciado. Loures (PT): Lusociência; 2006.

9. Ferreira MMC, Tavares J, Duarte J. Competências relacionais de ajuda nos estudantes de enfermagem. Referência: revista de educação e formação em enfermagem 2006 jun; II (2): 51-62.

10. Adam E. Ser enfermeira. Lisboa (PT): Instituto Piaget; 1994.

11. Queirós A. Empatia e respeito. Coimbra (PT): Quarteto Ed; 1999.

12. Sousa M. A formação contínua em enfermagem: estudo baseado nas perspectivas de enfermeiros. Nurs 2003 mar; 15 (175): 28-33.

13. Diener E, et al. The satisfaction with life scale. J Pers Asses 1985; 49 (1): 71-75.

14. Chalifour J. La relation d'aide eu soins infirmiers: une perspective holistique-humaniste. Montréal: Gäetan Morin Éditeur Itée; 1989.

15. Diener E, Suh ME. Subjective well-being and age: an international analysis. Annu Rev Gerontol Geriatr 1997; 17: 304-24. 